\title{
Fístula arteriovenosa renal secundaria a la ligadura en bloque del pedículo renal
}

\author{
Arzoz Fàbregas M*, Ibarz Servio L*, Bayona Areñas S*, Bernal Salguero S*, \\ Muchart Masaller $\mathrm{J}^{* *}$, Saladié Roig JM*. \\ *Servicio de Urología. **Sección de Radiología Vascular. Hospital Universitari Germans Trias i Pujol. Badalona. \\ Barcelona.
}

Actas Urol Esp. 2007;31(7):796-799

\section{RESUMEN}

FÍSTULA ARTERIO-VENOSA DEL PEDÍCULO RENAL DESPUÉS DE LA NEFRECTOMÍA

La fistula o aneurisma arteriovenoso del pedículo renal después de la nefrectomía es una complicación poco frecuente. La mayoría de las veces se diagnostica muchos años después de la cirugía.

Presentamos un caso de fistula arteriovenosa del pedículo renal derecho después de una nefrectomía realizada, por pionefrosis litiásica derecha, 40 años antes. Comentamos el cuadro clínico inicial, el tratamiento y la evolución del paciente. Se revisa la literatura actual.

Palabras clave: Fístula arteriovenosa. Pedículo renal. Nefrectomía.

\section{ABSTRACT}

ARTERIOVENOUS FISTULA OF THE RENAL STUMP AFTER NEPHRECTOMY

An arteriovenous fistula of the renal pedicle is a rare complication after nephrectomy. Most of cases we identify it time along after surgery.

We present a case of an arteriovenous fistula of the right renal pedicle after nephrectomy done for pyonephrosis 40 years before. We comment the clinical features, treatment and evolution of the patient. We also review the actual literature.

Keywords: Arteriovenous fistula. Renal hilium. Nephrectomy.

$\mathrm{E}^{\mathrm{s}}$ xisten tres tipos de fistula arteriovenosa renal (FAVR), las congénitas, las idiopáticas y las adquiridas. Las más frecuentes son las adquiridas, que constituyen el 75\% de las FAVR. Dentro de ellas, las más comunes son las secundarias a la biopsia percutánea renal, las producidas por traumatismo (ya sea penetrante o contuso) y las secundarias a cirugía percutánea renal, siendo una rareza las fistulas que aparecen después de una nefrectomía y más aun, las que se dan entre arteria renal y vena cava inferior ${ }^{1,2,3}$. Presentamos un caso de un paciente diagnosticado, 40 años después de la nefrectomía, de una fístula entre arteria renal y vena cava inferior.

\section{CASO CLÍnICo}

Paciente de 77 años, nefrectomizado hace 40 años por pionefrosis litiásica, que presenta un cuadro de dolor en flanco derecho continuo y clínica de disnea progresiva más edemas bimaleo- lares de un mes de evolución. En la exploración física, llama la atención la auscultación de un soplo continuo en el flanco derecho y en la radiografia de tórax, un aumento de tamaño de la silueta cardiaca y de ambos hilios pulmonares (Fig. 1). Se solicita de forma ambulatoria una Tomografía Axial Computerizada (TAC) con reconstrucción multiplanar de Angio-TAC, donde se observa una dilatación aneurismática del extremo distal de la arteria renal derecha de $3 \times 2$ $\mathrm{cm}$. de diámetro que comunica con la vena cava inferior (Fig. 2). Con el diagnóstico de fístula arteriovenosa del pedículo renal, se programa embolización percutánea de dicha fístula. Dos días antes de la embolización, el paciente acude a urgencias por episodio de insuficiencia cardiaca biventricular más fibrilación auricular de cronología incierta que obliga a iniciar tratamiento anticoagulante. Una vez estabilizado el cuadro de insuficiencia cardiaca, se realiza arteriografía 


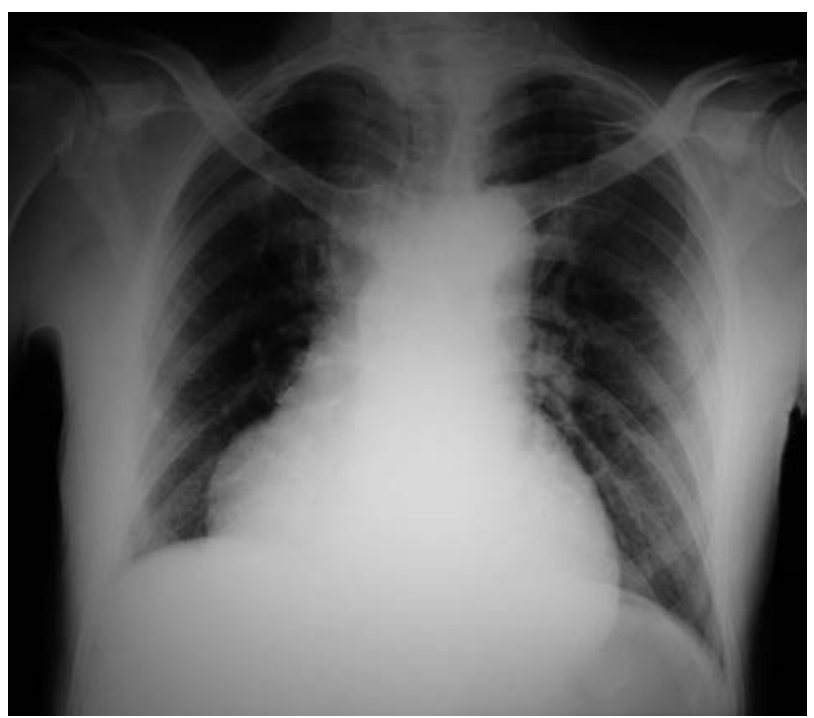

FIGURA 1. Radigrafía de Tórax: Aumento del tamaño de la silueta cardiaca y de ambos hilios pulmonares.

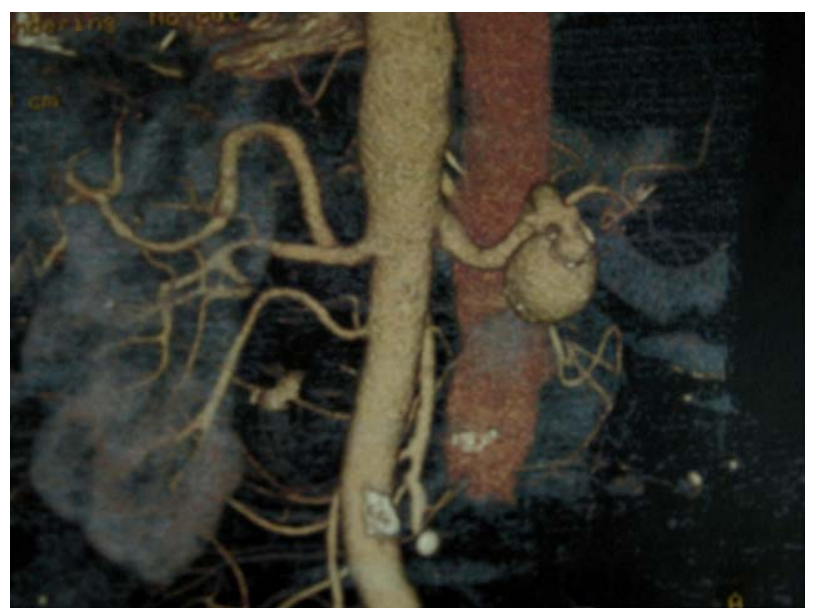

FIGURA 2. T.A.C abdominal con reconstrucción de AngioTAC (Postero-anterior): Dilatación aneurismática del extremo distal de la arteria renal derecha, de $3 \times 2 \mathrm{~cm}$ de diámetro, que comunica con la vena cava inferior.

renal derecha y embolización con espirales metálicas de la fistula, comprobando de forma inmediata la ausencia de comunicación arteriovenosa (Fig. 3 y 4). A las 24 horas el paciente estaba clínicamente asintomático. A los doce meses no se observa recidiva de la clínica.

\section{DISCUSIÓN}

La fístula o aneurisma arteriovenoso del pedículo renal después de la nefrectomía es una complicación poco frecuente. En la actualidad existen

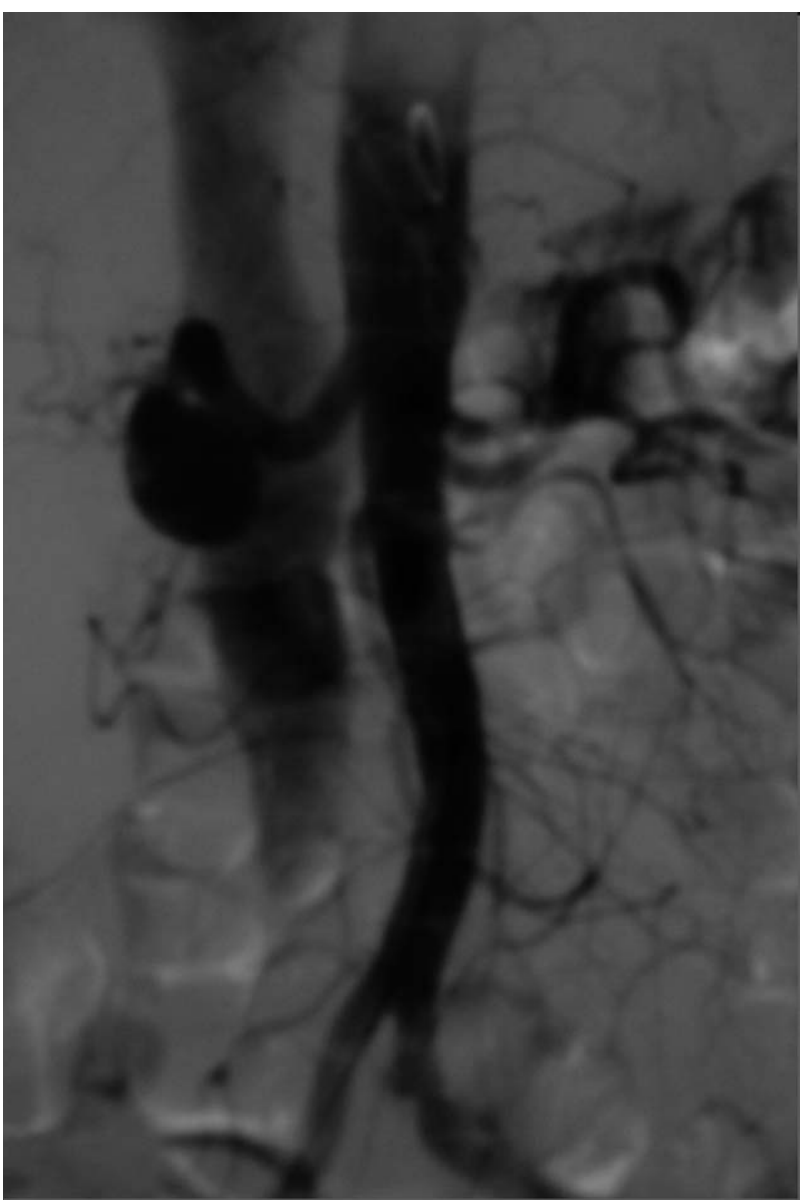

FIGURA 3. Arteriografia renal: Dilatación aneurismática de la arteria renal derecha que comunica con la vena cava inferior.

menos de 100 casos descritos en la literatura y menos de diez casos en el que la arteria renal comunique directamente con la vena cava inferior $^{1,4}$.

La creación de esta comunicación directa entre sistema arterial y venoso se atribuye al hecho de ligar en bloque el pedículo renal, con suturas de transfixión, favoreciendo así que las paredes venosas y arteriales, en contacto íntimo, se necrosen con la consecuente aparición de una fístula ${ }^{1,3}$. Otro factor de riesgo independiente para la creación de fístulas postnefrectomía, es la presencia de un proceso infeccioso, como tuberculosis o pionefrosis, en el momento de la nefrectomía, o bien a infecciones en el postoperatorio $^{1,2,4,5,6}$. A pesar de que la infección actúa como factor de riesgo independiente, se cree que el uso de ciertos materiales de sutura quirúrgico, espe- 


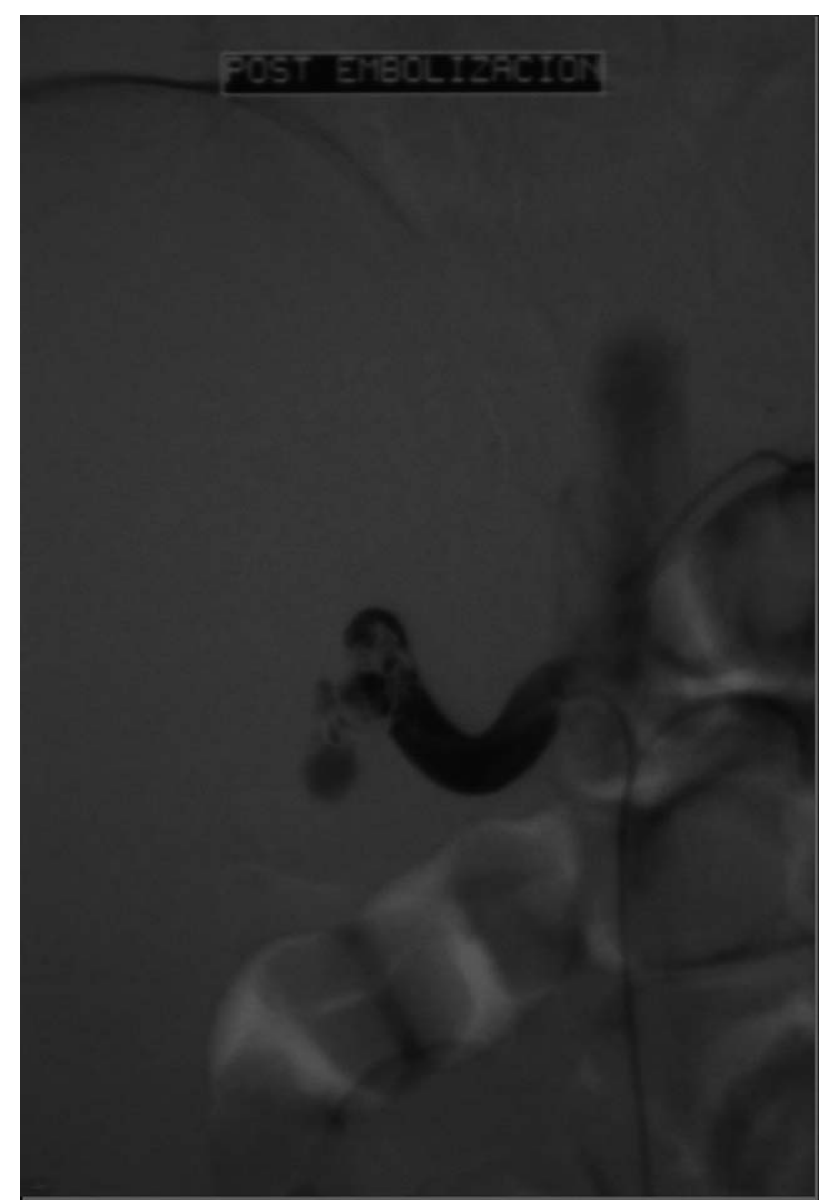

FIGURA 4. Arteriografía renal: Ausencia de comunicación arteriovenosa tras la embolización de la fistula con espirales metálicos

cialmente la seda trenzada, puede almacenar bacterias en aquellos casos que existe previamente un campo infectado ${ }^{6}$. En un 70\% de los casos se describen en el lado derecho, pues los vasos son más cortos en este lado y ello supone mayor dificultad en el momento de disecar los distintos componentes del pedículo para ligarlos por separado ${ }^{1,3,4}$. La comunicación del sistema arteriovenoso a nivel de la fístula produce un cortocircuito izquierda-derecha, con el consiguiente aumento del gasto cardíaco. Todo ello afecta a ambas cavidades ventriculares, con aparición de cardiomegalia y, a la larga, insuficiencia cardiaca congestiva $^{2,3}$.

La mayoría se diagnostican de forma tardía, muchos años después de la cirugía ${ }^{1,4}$, aunque existen casos aislados en los que se realizó el diagnóstico durante el primer año del postopera- torio $^{4,5}$. El tiempo medio desde la cirugía al diagnóstico se estima que es de 15 años ${ }^{1,3,5}$.

Las manifestaciones clínicas varian en función del tiempo entre la nefrectomía y el diagnóstico ${ }^{2,4}$. A pesar de que la presencia de un soplo continuo a nivel del flanco o del abdomen son diagnósticos, a menudo se sospechan ante un cuadro de hipertensión refractaria al tratamiento o por clínica de insuficiencia cardíaca congestiva, que es la complicación más importante de esta entidad y que suele aparecer en etapas tardías. En etapas iniciales, la presencia de una masa pulsátil abdominal, dolor en flanco o arritmias cardíacas, más el antecedente de nefrectomía, debería alertarnos de la posibilidad de este diagnóstico ${ }^{2,7}$. El diagnóstico precoz permite en ocasiones que los sintomas sean reversibles después del tratamiento ${ }^{1}$.

El diagnóstico de sospecha es básicamente clínico y, exploraciones como la ecografía doppler, la tomografía axial computerizada o sobretodo, la tomografía tridimensional, nos ayudan a confirmar el diagnóstico de sospecha. La arteriografía y en ocasiones también la tomografía tridimensional, es básica tanto para el diagnóstico como para estudiar la morfología de la fístula y decidir la mejor opción terapéutica ${ }^{4,7,8}$.

Todas las fístulas, y más aún las de gran calibre, deberían tratarse ante el riesgo de aparición de fallo cardíaco o, en menos ocasiones, de ruptura espontánea del aneurisma ${ }^{4,9}$. El tratamiento de elección depende del tamaño y de la etiología de la fístula. La exéresis completa de la fístula, la ligadura simple de la vena y arteria o la embolización percutánea, son medidas terapéuticas que han demostrado ser eficaces para el tratamiento de esta patología ${ }^{4,9}$. En fístulas sintomáticas y de pequeño tamaño, la embolización percutánea es el tratamiento de elección, reservando la cirugía de exéresis para las fístulas arteriovenosas de gran tamaño no resueltas con embolización y la ligadura simple para los casos de fístula de gran tamaño en que la exéresis es dificultosa debido a la inflamación de alrededor ${ }^{4}$.

La embolización percutánea, aunque mucho más ventajosa respeto a la cirugía abierta, no está libre de riesgos. Se han descrito casos de migración de los agentes embolizantes hacia el sistema circulatorio pulmonar (sobre todo en fís- 
tulas de gran tamaño), casos de isquemia intestinal o renal contralateral y casos de reaparición de la fístula. En la literatura se describen distintas técnicas de embolización percutánea, en las que se usan materiales diversos, con la intención de disminuir el riesgo de migración ${ }^{4,9,10}$.

La cirugía de exéresis consiste en individualizar la lesión, con ligadura por separado de arteria y vena, previa resección del trayecto fistuloso. En los casos de exéresis dificultosa, la ligadura simple de los vasos puede dar buenos resultados, aunque el riesgo de recidiva es mayor ${ }^{4,11}$. La indicación de tratamiento de esta patología es absoluta, pues tanto la embolización percutánea como la cirugía, solas o de forma combinada, son técnicas que permiten resolver estas fístulas de forma eficaz, evitando la aparición de insuficiencia cardiaca o resolviéndola en los casos de evolución temprana ${ }^{1,3,9,10}$.

\section{CONCLUSIÓN}

Aunque es una entidad poco frecuente, la presencia de una fístula arteriovenosa del pedículo renal después de una nefrectomía, es una patología que debemos sospechar ante síntomas de insuficiencia cardiaca o soplos abdominales junto al antecedente de la nefrectomía. Disponemos de la embolización percutánea como tratamiento menos invasivo y muchas veces curativo, y el hecho de tratarlas de forma precoz, en el momento del diagnóstico, permite evitar graves consecuencias a largo plazo.

\section{REFERENCIAS}

1. Ferrari M; Bonanomi G; Catalana G; Cioni R, Mosca F. Combined percutaneous surgical approach to a postnephrectomy arteriovenous fistula. J Cardiovasc Surg 2001; 42(3):393395.

2. Kajbafzadeh AM, Broumand B. Arteriovenous fistula following Nephrectomy. Eur Urol.1997; 31(1):112-114.

3. Bedós F, Cibert J. Aneurisma arteriovenoso. Bedós F, Cibert J, editores. Urología. La terapéutica y sus bases. Urologia. Barcelona. Espax. 1989, pp 77-82.

4 .Matos A, Moreira A, Mendoza M. Renal arteriovenous fistula after nephrectomy. Ann Vasc Surg 1992; 6:378-380.

5. Lacombe M. Renal arteriovenous fistula following nephrectomy. Urology 1985; 25:13-16.

6. Rapp DE, Orvieto M.A, Gerber G.S, Johnston W.K, Wolf J.S, Shalhav A.L. En Bloc stapling of renal hilium during laparoscopic nephrectomy and nephroureterectomy. Urology. 2004; 64(4):655-659.

7. Okamoto M, Hashimoto M, Akita T, Sueda T, Karakawa S, Ohishi Y, et al. Congestive heart failure caused by aortocaval fistula after nephrectomy. Intern Med. 2001; 40(11):11131116.

8. Ozaki K, Kubo T, Hanayama N, Hatada K, Shinagawa H, Maeba $\mathrm{S}$, et al. High-output heart failure caused by arteriovenous fistula long after nephrectomy. Heart Vessels.2005; 20(5).236238.

9. Kocakoc E, Poyraz A.K, Cetinkaya Z, Bozgeyik Z. Postnephrectomy renal arteriovenous fistula. J Ultrasound Med.2004; 23(7):965-968.

10. Resnick S, Chiang A. Transcatheter embolization of a high-flow renal arteriovenous fistula with use of constrained wallstent to prevent coll migration. Vasc Interv Radiol. 2006(2 pt1);17:363367.

11. Hirai S, Hamanaka Y, Mitsui N; Kumagai H, Nakamae N. Highoutput heart failure caused by a huge renal arteriovenous fistula after nephrectomy: report of a case.Surg Today.2001;31 (5):468-470.

Correspondencia autor: Dra. Montserrat Arzoz Fàbregas Servicio de Urología. Hospital Universitari Germans Trias i Pujol. Badalona. Ctra. del Canyet, s/n - 08916 Badalona (Barcelona) Tel.: 934651200

E-mail autor: 37031maf@comb.es Información artículo: Nota Clínica Trabajo recibido: julio 2006

Trabajo aceptado: octubre 2007 